Michele Cutino

\title{
Fictions poétiques et vérités bibliques dans les réécritures vétéro et néotestamentaires en vers
}

\section{Questions méthodologiques}

En commençant cette contribution à ce colloque, qui, je l'espère, sera riche de discussions et fécond en résultats, il me semble nécessaire d'éclaircir la perspective précise de ma contribution dédiée aux questions méthodologiques concernant le rapport entre fictions poétiques et vérités bibliques dans les paraphrases vétéro et néotestamentaires. Si l'on devait s'en tenir aux déclarations programmatiques de ces poètes, il n'y aurait rien à approfondir. En effet, si l'on considère les affirmations explicites de ces auteurs, il va de soi qu'ils soulignent comment l'horizon de leur inspiration est la vérité historique de la Bible: ces poèmes sont véritables, comme l'affirme Arator dans l'épitre-préambule de son Historia Apostolica adressée à Virgile $^{1}$, parce qu'ils suivent l'hypotexte biblique, ici les Actes des Apôtres de Luc, qui est histoire. Et il va également de soi que cette vérité historique est constamment opposée dans ces déclarations aux fictions poétiques de la poésie païenne: tout le monde sait bien comment, tout au début de leurs poèmes sur l'évangile, Juvencus dans ses Evangeliorum libri ${ }^{2}$ et Sedulius dans son Paschale Carmen ${ }^{3}$, soulignent que les Christi vitalia gesta pour Juvencus, et les clara salutiferi miracula Christi pour Sedulius, sont d'autant plus dignes d'être célébrés en vers que, comme l'observe Juvencus, des poèmes comme ceux d'Homère et de Virgile ont connu une réputation durable, alors qu'ils entremêlent de mensonges la geste des hommes anciens, ou que, comme le fait remarquer Sedulius, les poètes gentils font un étalage grandiloquent de leurs fictions ${ }^{4}$. Sans parler de la prise de position nette sur cette question, au moins sur le plan théorique, de la part du poète vétérotestamentaire Avit, qui, dans la lettre/dédicace de ses livres De spiritalis historiae gestis à Apollinaire ${ }^{5}$, in-

1 Arat. ad Vigil. 19-20 : Versibus ergo canam quos Lucas rettulit Actus, / historiamque sequens carmina vera loquar.

2 Iuvenc. praef. 15-18: quae veterum gestis hominum mendacia nectunt, nobis certa fides aeterna in saecula laudis inmortale decus tribuet meritumque rependet. Nam mihi carmen erit Christi vitalia gesta.

3 Sedul. Pasch. carm. I.17-18; 23; 26 : Cum sua gentiles studeant figmenta poetae / grandisonis pompare modis ... cur ego ... clara salutiferi taceam miracula Christi?

4 Pour les rapports entre la préface des Evangeliorum libri de Juvencus et celle du Carmen Paschale de Sedulius je renvoie à la contribution de Costanza (1985) 253-286.

5 Avit. epist. ad Apol. : Quamquam quilibet acer ille doctusque sit, si religionis propositae stilum non minus fidei quam metri lege servaverit, vix aptus esse poemati queat; quippe cum licentia mentiendi, 
terdit radicalement la licentia mentiendi, la permission de dire des mensonges, quand on traite d'arguments sérieux comme les matériels bibliques, alors qu'Horace dans son Art poétique ${ }^{6}$ avait accordé cette liberté aux poètes et aux peintres: et il dit expressément qu'il ne s'agit pas seulement ici des fictions du mythe, mais des mensonges poétiques en général ${ }^{7}$.

Reste également en dehors de la perspective de mon intervention l'ambiguïté avec laquelle les poètes chrétiens, en dépit de leurs déclarations poétiques éloquentes, s'approchent de l'imaginaire de la poésie profane, jugée viciée par le mensonge, aspect que Paul Augustin Deproost ${ }^{8}$ a mis bien en évidence dans sa contribution sur le rapport entre ficta et facta dans la poésie chrétienne latine. C'est une ambiguïté qui ressort de toute évidence du fait que les poètes chrétiens trouvent dans le langage de la poésie païenne, notamment dans les images de la fabula épique, des vérités cachées qui les intéressent, ainsi que les moyens expressifs privilégiés pour communiquer ces vérités, si bien qu'on peut parler, à cet égard, d'un véritable double langage.

Notre intervention porte plutôt sur une troisième perspective, plus rare, mais, je pense, très intéressante, c'est-à-dire l'insertion consciente de la fiction littéraire dans le récit biblique là où celui-ci est, de quelque façon, incomplet, ou, ce qui est encore plus radical, la substitution de la fiction poétique à l'histoire biblique qui subit, par conséquent, une ré-sémantisation profonde.

La première de ces possibilités, je l'ai mise en évidence à propos du poème Alethia composé par Claudius Marius Victorius selon toute probabilité dans le deuxième quart du $\mathrm{V}^{\text {ème }} \mathrm{s}$., dans la monographie que je lui ai consacrée en $2009^{9}$, dont je vais reprendre et approfondir quelques données. En effet, au début du deuxième livre de ce poème (vv. 1-5), nous trouvons un bref préambule de cinq vers, introduits selon des formules de récapitulation typiques des poèmes didactiques :

Hactenus arcanam seriem, primordia mundi,

ut sincera fides patuit, sine fraude cucurri,

dum dignis leto vitiis terrena carerent.

Nunc hominum mores et iam mortalia versu

ingressum fas sit veris miscere poetam.

«Jusqu'ici j’ai parcouru, sans aucun mensonge, d'après la manifestation de la foi sincère, la succession mystérieuse des événements et des origines du monde, pendant que la réalité ter-

quae pictoribus ac poetis aeque conceditur [Hor. ars 9], satis procul a causarum serietate pellenda sit. In saeculari namque versuum opere condendo tanto quis peritior appellatur, quanto elegantius, immo, ut vere dicamus, ineptius falsa texuerit. Taceo iam verba illa vel nomina, quae nobis nec in alienis quidem operibus frequentare, ne dicam in nostris conscribere licet: quae ad compendia poetarum aliud ex alio significantia plurimum valent.

6 Hor. Ars 9-10: Pictoribus atque poetis / quidlibet audendi semper fuit aequa potestas.

7 Sur ce texte voir Roberts (1980) en particulier 400-402.

8 Deproost (1998) 101-121.

9 Cutino (2009). 
restre échappait aux vices dignes de la mort. Maintenant que j’ai commencé à traiter en vers les coutumes des hommes et les réalités désormais mortelles, qu'on m'accorde de mêler l'attitude du poète à la vérité» (trad. M. Cutino)

Ce préambule constitue un important tournant de la stratégie de composition du poème, comme on peut le remarquer du fait que Victorius reprend consciemment une déclaration programmatique de Paulin de Nole dans le Natalicium $12^{10}$-nous suivons la numérotation de la nouvelle édition des poèmes de cet auteur par Fr. Dolveck (CCSL 21, 2015) - déclaration qui porte justement sur le rapport entre vérités chrétiennes et fictions poétiques. Dans ce passage, Paulin revendiquait comme point fort du lettré chrétien, le fait que, même s'il compose des poèmes, il ne recourt jamais à la fraus poetae, c'est-à-dire aux faussetés qui sont propres aux poètes païens : cette fraus, en effet, peut intéresser selon Paulin seulement les gentils, dévoués aux pratiques idolâtriques, non les chrétiens, qui, en ayant comme mesure de leur propre production poétique et artistique la foi en Jésus Christ, se fondent sur l'historicité de cette foi qui caractérise leurs récits, relatifs dans ce poème à trois miracles animaliers œuvrés par les reliques de saint Félix à Nole.

La reprise antiphrastique des affirmations pauliniennes est ultérieurement enrichie et renforcée par l'allusion à un passage de l'Art poétique d'Horace concernant la licentia mentiendi que, comme nous l'avons dit, Avit-à mon avis en référence allusivement polémique à ce qu'affirme justement Victorius et à ses modalités de narration dans ce deuxième livre ${ }^{11}$ - avait condamnée également en rapport à un passage de ce texte d'Horace : en effet, dans les vv. $149-152^{12}$ le poète classique fait l'éloge d'Homère, capable d'imaginer si bien ses mensonges, et de mêler si bien invention/fausseté et vérité/réalité qu'il confère une unité profonde à son ouvrage du début à la fin en passant par le milieu. Il est évident que l'hémistiche veris miscere poetam du v. 5 d'Alethia II est calqué sur l'hémistiche du v. 152 d'Horace sic veris falsa remiscet, avec l'importante substitution de falsa avec poeta, employé avec valeur antonomastique pour poésie, attitude des poètes. Substitution qui fait remarquer que le poète ne peut pas ne pas dire de mensonges. Et Victorius demande-fas sitjustement cette licence dans son préambule. Il y a de quoi s'étonner, parce que le bouleversement du modèle paulinien est conduit à l'intérieur d'un poème biblique, c'est-à-dire d'un genre littéraire qui est basé de façon programmatique, comme nous venons de le voir, sur la vérité historique.

Cependant, cette licence est demandée par Victorius par rapport au contenu qu'il va traiter : il s'agit des mœurs des hommes, devenus mortels, après la faute des

10 Paul. Nol. Nat. 12.28-32 : Non adficta canam, licet arte poematis utar. / Historica narrabo fide sine fraude poetae: / absit enim famulo Christi mentita profari. / Gentibus hae placeant ut falsa colentibus artes; / at nobis ars una fides et musica Christus.

11 Cutino (2009) 221-224.

12 Hor. ars 151-152 : atque ita mentitur, sic veris falsa remiscet, / primo ne medium, medio ne discrepet imum. 
protoparents Adam et Ève. C'est pourquoi ce sujet exige des formes expressives différentes par rapport à celles utilisées jusqu'ici par lui : si en effet il avait traité les primordia mundi, c'est-à-dire Gen 1-3 sine fraude, parce que dans ceux-ci s'était manifestée la foi sincère, cette fois s'étant corrompue à cause du péché originel, il y a l'exigence de modifier aussi le code stylistique. Victorius théorise, donc, la possibilité de recourir aux mensonges pour un poète-tout en sachant bien qu'il s'agit de mensonges-pour mieux illustrer la décadence de l'humanité dans la condition postlapsaire. Ça veut dire que le mensonge n'est pas autorisé dans l'absolu, mais dans le cadre d'un critère précis d'homologie entre forme et contenu. Il est important, en effet, de remarquer, à cet égard, qu'après cette ouverture programmatique le livre II du v. 6 jusqu'au v. 195 est occupé par une digression sur les modalités à travers lesquelles Adam et Eve chassés de l'Eden, se sont adaptés à la nouvelle situation caractérisée par le besoin, en découvrant par hasard le feu et les métaux : l'intertexte de cette digression est constitué, comme je l'ai souligné dans la dite monographie, par la conclusion du livre V du De rerum natura concernant la condition des hommes primitifs et leur évolution progressive. Par ailleurs, le récit de ces découvertes dans Alethia II se conclut (vv. 179-185) avec la prosopopée de Dieu même qui s'oppose au rôle du hasard et de l'expérience dans la connaissance humaine, ressortant du modèle lucrétien, en revendiquant les dons qu'il a fait providentiellement à l'homme de la connaissance innée. A la fin de cette digression, en tout cas, Victorius, en se référant très librement à Gen 4.1 «Adam s'unit à Eve, sa femme, et elle conçut et enfanta Caïn», se hâte de préciser déjà à partir du v. $195^{13}$ qu'on peut, en tout cas, s'en tenir aux limites de la vérité biblique, consistant dans le fait que le premier intérêt témoigné par Adam ayant quitté le paradis, fut justement l'amour pour sa femme et les enfants qu'elle lui donna.

Le critère donc de l'homologie et le recours à l'une des modalités narratives typiques des réécritures de la bible en vers, comme la digression/excursus, mettent, de quelque façon, en sourdine l'insertion dans la narration biblique, de l'un des thèmes pratiqués et aimés par la poésie profane, celui du progrès de l'histoire humaine à partir de sa condition primitive.

Ces cautèles, ces précautions sont tout à fait absentes dans un autre cas, cette fois concernant une paraphrase néotestamentaire, que je veux prendre en considération de plus près. Il s'agit d'un passage de la Laus Iohannis, petit poème dédié à la figure de Jean Baptiste, attribué, pour ainsi dire, jusqu'à hier à Paulin de Nole, mais non reproduit dans la dernière édition, déjà mentionnée, des poèmes de cet auteur par Fr. Dolveck, avant tout parce que dans l'intitulatio du manuscrit qui nous a transmis intégralement l'œuvre, manque l'indication de l'auteur. Sans entrer dans cette question complexe-que A.-M. Turcan-Verkerk ${ }^{14}$ a le mérite d'avoir posée et dont je suis en

13 Mar. Vict. Aleth. II.195-198 : ... Summo ergo hoc limite veri / haereat, augustis quod pulsus sedibus Adam, / nullum aliud prius officium quam coniuge dignum / egit et ignotos natis impendit amores.

14 Turcan-Verkerk (2003) en particulier 155-164. 
train de m'occuper, - le passage que je prends en considération est celui des vv. 229254 qui est articulé autour de la description des mœurs de Jean Baptiste pendant sa retraite dans le désert, dont l'intertexte biblique est Mc 1.6 et Mt $3.4^{15}$ :

Vestis erat curvi saetis conserta cameli

contra luxuriem molles duraret ut artus

arceretque graves conpucto corpore somnos.

Hunc vilis rigidos ad lumbos zona ligabat.

Praebebant victum facilem silvestria mella

pomaque et incultis enatae cautibus herbae

235 arentemque sitim decurrens unda levabat.

Quis locus hic vitiis? Aditum quem prava cupido

invenit haec inter sacrae ad penetralia mentis?

Quo peccet qui nil cupiat? Quo tendat iniqui

illecebras sensus quisquis non indiget ullo?

240 Sic primi vixere homines, mundoque recenti

hos auctor dederat ventura in saecula mores, inseruit donec sese malesuada voluptas

ac secum luxus et amorem invexit habendi.

Hinc odia, hinc lites, hinc fraus, hinc livor et irae,

245 caedes arma cruor conflictus proelia mortes,

hinc offensa dei, quam tartara saeva piabunt.

Verum ego cur nimium communes arguo culpas,

inmemor ipse mei, quem non commissa gravare, sed veniam sperare decet? Mirabimur immo

250

rectius invictum nullique imitabile prisci

exemplum saecli transgressum humana labore

semideumque virum, qui labe inmunis ab omni,

cum sua tam saevis cruciarit corpora poenis,

praescripsit quid nos vel post peccata deceret ${ }^{16}$.

«Son vêtement était tissu des poils d'un chameau courbé, pour endurcir les membres amollis contre le luxe et empêcher le sommeil lourd en piquant le corps. Il était attaché à ses hanches rigides une ceinture humble. Miel sauvage, fruits et herbes poussées sur des rochers incultes lui fournissaient une nourriture aisée, et l'eau courante étanchait sa soif ardente. Quelle place dans cette condition pour les vices ? Comment, dans ces réalités, la convoitise perverse pourrait-elle avoir accès à l'intérieur d'un esprit consacré ? En quoi pourrait pécher qui n’a aucun désir ? Où pourrait diriger les tentations d'un sentiment malveillant celui qui n’a besoin de rien ? Ainsi vécurent les premiers hommes, selon ces coutumes que le créateur avait données pour les siècles futurs au monde qui venait d'être créé, jusqu'à ce que le plaisir, mauvais conseiller, ne s’y

15 Mc 1.6 : Et erat Iohannes vestitus pilis cameli / et zona pellicia circa lumbos eius, et lucustas et mel sivestre edebat; Mt 3.4 : Ipse autem Iohannes habebat vestimentum de pilis camelorum et zonam pelliciam circa lumbos suos. Esca autem eius erat lucustae et mel silvestre. Il s'agit de l'une des intégrations - par ailleurs, très peu nombreuses -à l'intertexte fondamental de ce poème paraphrastique qui est constitué par Lc $1 ; 3 ; 7$.

16 Le texte suit l'édition critique que je vais préparer de cet ouvrage: en particulier, je fais remarquer au v. 239 la leçon illecebras que ja’i choisie à la place de in latebras de la dernière édition révisée (par M. Kamptner) de von Hartel dans CSEL 30, Vindobonae $1999^{2}$. 
insinuât et n'apportât avec lui la luxure et l'amour pour les richesses. D'où vinrent les haines, d'où les litiges, d'où la tromperie, d'où l'envie et la colère, les massacres, les armes, le sang, les conflits, les batailles, les morts, d'où l'offense envers Dieu, qui sera expiée par le Tartare cruel. Mais pourquoi est-ce que je reproche trop les fautes communes, m'oubliant moi-même, alors qu'il me convient non d'aggraver ce que j'ai commis, mais d'espérer le pardon ? Au contraire, il sera plus juste d'admirer cet exemple du temps passé invaincu et inimitable, qui a dépassé par ses efforts les possibilités humaines, et cet homme demi-dieu, qui, exempt de toute tache, ayant crucifié son corps à travers des supplices si cruels, nous a prescrit ce qu'il convient de faire même après les péchés». (trad. M. Cutino)

A propos de ce passage on a déjà remarqué - en particulier P. Flury ${ }^{17}$ et A.V. Nazzaro ${ }^{18}$ -la dette de l'auteur envers la description de Baptiste par Juvencus ${ }^{19}$ et la présence dans la description de ses mœurs des particularités appartenant au motif littéraire de l'âge d'or, notamment des Saturnia regna dont parle Virgile à plusieurs reprises dans Géorgiques II et dans Énéide VIII. En effet les vv. 229 (Vestis erat curvi saetis conserta cameli) et 232-233 évoquent sans aucun doute, surtout ce dernier v. 233, le modèle de Juvencus, tout comme il est évident que l'insertion dans ce même v. 233 de facilem à la place de tenuem (victum) de Juvencus donne lieu à une tournure propre à la caractérisation de la vie heureuse des agriculteurs, auxquels la terre fournit un aliment «facile», vie heureuse que dans le texte de Géorgiques II Virgile rapproche de la condition également bienheureuse des hommes à l'époque de Saturne, avant que la Justice quittât la terre ${ }^{20}$. Ces références méritent d'être approfondies à travers une analyse attentive du contexte dans lequel s'insère cette présentation de Baptiste, qui donne matière à d'importantes réflexions à propos de l'interaction entre vérités bibliques et faussetés poétiques. Remarquons avant tout que la particularité du manteau en poils de chameau et du pagne de peau autour des reins portés par Jean Baptiste, enregistrée fidèlement par Juvencus, dans la Laus Iohannis est amplifiée : pour l'auteur ce vêtement est conçu comme un véritable antidote contre le luxe, afin d'endurcir les membres mouillés, si bien que les reins de Baptiste sont définis au v. 232 rigides, rigidos lumbos, et afin de repousser le sommeil lourd au moyen des poils piquant son corps. C'est un élément qui renvoie sans aucun doute au milieu monastique : on le retrouve, en effet, dans la transposition en vers de la Vita Martini de Sulpice Sévère par Pauline de Périgueux à propos de la praxis de certains aristocrates qui à Marmoutier se conformaient à cette typologie exotique de vêtement, rapportée par les pèlerins d'Orient, pour mortifier leur chair dans leurs surenchères ascéti-

17 Flury (1973) 129-145. Cette étude est encore l'unique expressément consacrée à l'analyse formelle globale de la Laus.

18 Nazzaro (2003) 283-306 ; Nazzaro (2004) 19-33 ; Nazzaro (2006) 133-146 ; Nazzaro (2008) 315324.

19 Iuvenc. evang. I.323-325 : Texta camelorum fuerant velamina saetis, / et zonae pellis medium cinxere profetam / et tenuem victum praebent silvestria mella.

20 Verg. georg. II.458-460 : O fortunatos nimium, sua si bona norint, / agricolas! Quibus ipsa procul discordibus armis / fundit humo facilem victum iustissima tellus. 
ques $^{21}$. Mais la référence à l'endurcissement voulu des membres rigides nous conduit plutôt à la récupération de la condition primordiale des hommes avant que le luxe alanguît le corps à l'origine résistant et fort, comme le soulignent Lucrèce dans sa digression, déjà mentionnée, sur les hommes primitifs à la fin du $\mathrm{V}^{\mathrm{e}}$ livre de son $D e$ rerum natura ${ }^{22}$, et ensuite Prudence dans son Hamartigénie dans un passage où nous trouvons rigidos duraverat artus référé aux intentions, au projet du créateur sur l'homme avant sa corruption par le luxe $\mathrm{e}^{23}$.

Le modèle de Juvencus subit des modifications/amplifications à propos aussi de la nourriture du Baptiste qui vont dans le même sens : avant tout, il est vrai que le facilis victus de v. 233 rappelle georg. II, mais il ne faut pas également oublier le réemploi de cette tournure par Juvencus dans la paraphrase de Mt $6.24-25^{24}$, là où le Christ exhorte ses disciples, sur le modèle des oiseaux qui sont facilement alimentés par la terre, à ne pas se tourmenter avec des questions de nourriture ou de vêtement : le facilis victus évoque ici la disponibilité immédiate des dons de la nature créée par Dieu. Ensuite, dans la Laus, en dépit de l'omission par Juvencus des sauterelles dans le régime alimentaire du prophète, omission suivie par notre auteur, nous avons la mention des fruits, dans la forme coordonnée au début du v. 234 pomaque, qui rappelle un texte des Amours d'Ovide ${ }^{25}$ concernant encore la nourriture spontanée fournie par la nature dans l'âge d'or. L'image des poma/fruits, par ailleurs, étant associée dans ce vers aux herbes non cultivées, nées du terrain rocheux, n'évoque pas du tout le caractère luxuriant de la nature, propres à certaines descriptions de

21 Paul. Petr. Mart. II.141-148 : Multis vestis erat saetis contexta cameli, / quae levibus stimulis vigiles contingeret artus, / excludens tenuem compuncta carne soporem, / mollia ne fluxum nutrirent vellera corpus / lasciva et tenui vestis fluitaret amictu, / qualia praeteritae consuerat copia vitae. / Nam multos illuc prisco de sanguine claros traxerat ad talem prudens sententia vitam : pour ce rapprochement voir Weidmann (1991) 174-175. L'hypotexte de référence est Sulp. Sev. Mart. 10, 8 (plerique camelorum saetis vestiebantur: mollior ibi habitus pro crimine erat), où on parle de l'habitude d'une grand nombre de disciples de Martin dans l'anachorèse, de porter justemement des habits de poils de chameau, croyant être un crime de se vêtir plus délicatement.

22 Lucr. V.925-938: At genus humanum multo fuit illud in arvis / durius, ut decuit, tellus quod dura creasset, / et maioribus et solidis magis ossibus intus / fundatum.

23 Prud. ham. 279 ; 282-284 : Quid quod et ipse, caput muliebris corporis et rex, / ... / solvitur in luxum? Cernas mollescere cultu / heroas vetulos, opifex quibus aspera membra / finxerat et rigidos duraverat ossibus artus. Voir, à cet égard, Nazzaro (2008) 318 n. 11.

24 Iuvenc. evang. I.631-633 : Neu faciles victus aegra disquirite cura. / Nonne animam pluris facimus quam corporis escas / aut dubitat corpus quisquam praeponere vesti?

25 Ov. am. III.8.33-34: At meliora dabat curvo sine vomere fruges / pomaque et in quercu mella reperta cava, où on peut remarquer également l'association entre mel et poma. L'absence des sauterelles depend avant tout du modèle de Juvencus, mais il est clair qu'elle produit un portrait 'végétarien' de l'alimentation du Baptiste qu'on a rattaché à l'influence du modèle de l'Adversus Iovinianum de Jérôme: cf. Duval (1989) 184-187. 
l'âge d'or ${ }^{26}$, mais insiste plutôt sur la frugalité et l'essentialité de cette nourriture spontanée, comme le faisaient les textes illustrant la condition de vie des hommes primitifs, en particulier le déjà cité livre V du De rerum natura ${ }^{27}$. Mais c'est surtout le dernier élément de cette description, tout à fait gratuit par rapport à l'hypotexte biblique, qui est très intéressant : Jean Baptiste, affirme notre auteur, satisfaisait la soif en buvant l'eau courante d'un fleuve : cet élément évoque sans aucun doute l'une des conditions des hommes primitifs encore selon le modèle du De rerum natura $\mathrm{V}^{28}$.

Si l'on met ensemble ces éléments il est évident que l'auteur de la Laus à travers les allusions intertextuelles, représente Baptiste comme suivant le régime de vie des premiers hommes, et que pour lui l'essentialité, l'autarkeia de ce régime constitue le véritable âge d'or de l'humanité. Ainsi il peut conclure, à la fin de cette présentation, aux vv. 240 - 241 que les premiers hommes vécurent ainsi (sic primi vixere homines) et que Dieu avait prévu cette condition pour le temps à venir (hos auctor dederat ventura in saecula mores), deux vers qui sont encore une adaptation savante de passages de la poésie païenne concernant l'âge d'or ${ }^{29}$.

Jusqu'ici il n'y a rien d'étonnant, on reste dans le réemploi des matériels littéraires païens dans la réécriture biblique pour des finalités stylistiques, expressives. C’est la suite qui revêt un intérêt particulier pour notre sujet, c'est-à-dire la présentation de la perte de la part de l'humanité corrompue de cette condition édénique, basée sur l'autarkeia, et de ses conséquences sur le plan théologique. À cet égard, il me plait de mettre au clair le développement argumentatif à travers un schéma (Fig. 1).

La corruption de l'humanité est décrite selon un réemploi original du motif païen de la diffusion des maux dans le monde suite à la fin de l'âge d'or et à la succession d'âges progressivement inférieurs par rapport à celui d'or, jusqu'à l'âge de fer. L'originalité consiste avant tout dans le fait que ce motif se substitue complètement aux références à l'histoire biblique de cette dégradation à partir du péché des protoparents. Ce péché est sans doute évoqué par la mention du premier vice qui est à l’origine de la décadence, la malesuada voluptas du v. 242, mais il s'agit d'une évocation allusive et en tout cas à travers la médiation exégétique. En effet, l'unique intertexte biblique possible, le suasit de certaines traductions Veterae latinae, relatif

26 C'est pourquoi je ne suis pas d'accord avec ceux qui voient dans la description de cette condition une sorte de scène idyllique : cf. Nazzaro (2008) 323 n. 32 qui renvoie à Lupieri (1984) 48-49 qui fait rentrer ce paysage dans l' 'idéologie claustrale' du monachisme de Lérins.

27 Lucr. V.937-938: Quod sol atque imbres dederant, quod terra crearat, / sponte sua, satis id placabat pectora donum.

28 Lucr. V.945: At sedare sitim fluvii fontesque vocabant. Je me réfère au concept, parce que sur le plan formel, comme l'a bien remarqué Nazzaro (2008) 319 n. 15, le vers rappelle Ov. epist. 4.174 : arentem quae levet unda sitim.

29 Cf. Eleg. in Maec. 1.23-24 : Sic illi vixere quibus fuit aurea Virgo, / quae bene praecinctos postmodo pulsa fugit ; Avien. Arat. 292-294 : ... tu cum sincera priorum / vita hominum nullis ageret sub legibus aevum / aureaque innocuos servarent saecula mores. 
vv. $229-241$

Présentation orientée du récit évangélique (Mc 1.6 ; Mt 3.4)

sur la nourriture et sur le vêtement de Jean Baptiste :

celui-ci vit selon la condition de frugalité et d'essentialité

voulue par Dieu pour les premiers hommes,

véritable âge d'or de l'histoire humaine

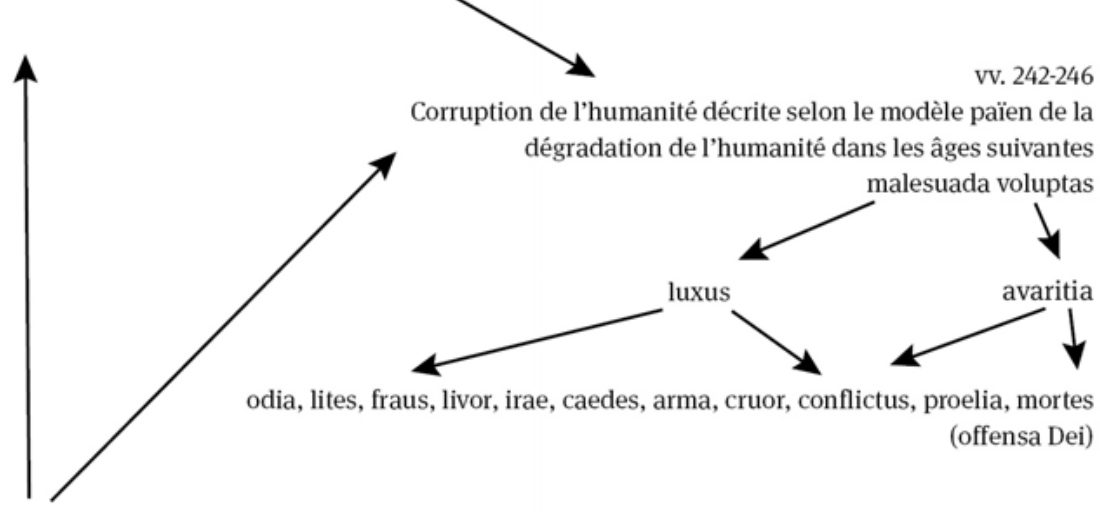

vV. $247-254$

Interprétation théologique : Jean Baptiste, étant un renouveau exceptionnel, hérö̈que, dans des temps de corruption, du modèle de la vie primitive bienheureuse, ignorant du péché, fournit un modèle actuel aux hommes : au lieu de se concentrer sur la condition pécheresse de toute l'humanité, il les exhorte à poursuivre un idéal de vie convenant même après les péchés commis.

Fig. 1

à la justification qu'Eve donne de son péché devant Dieu, en l'attribuant à la tentation du serpent/diable, est englobé dans l'adjectif poétique malesuada d'allure déjà virgilienne-la très fameuse malesuada fames du vestibule des Enfers dans l'Énéide (VI.883)- et il est associé au substantif abstrait voluptas selon l'identification du péché des protoparents avec le plaisir, origine de tous les autres maux, proposée par les exégètes chrétiens, avant tout par Ambroise ${ }^{30}$. Mais après disparaissent même des allusions possibles au canevas biblique : de la voluptas viennent des maux, le luxe et l'avidité, dont la complémentarité dans l'imaginaire classique comme causes

30 Ambr. parad. 15.73 : «Serpens» inquit «suasit» (Gen 3.13) ... Serpens enim mulierem decepit , virum mulier ad praevaricationem de veritate deduxit. Serpentis typum accepit delectatio corporalis. Mulieris symbolum sensus est nostri, vir mentis. Delectatio itaque sensum movet, sensus menti transfundit quam accepit passionem. Delectatio igitur prima est origo peccati. 
principales de la dégradation de l'humanité est topique ${ }^{31}$. Et observons que l'avidité en particulier est évoquée à travers une tournure expressive, amor habendi, appartenant à la réécriture virgilienne de la fin de l'âge d'or dans le huitième livre de l'Enéide ${ }^{32}$. De ces deux maux, ainsi, viennent tous les autres selon une liste dans les vv. 244-245 qui appartient elle-même à la topique des maux apportés à l'humanité par les âges suivant celui d'or- la référence formelle la plus proche c'est celle des Métamorphoses d'Ovide ${ }^{33}$. Le module expressif de la liste des maux a séduit les poètes chrétiens mais elle n'est jamais utilisée, comme dans notre cas, pour connoter la dégradation de l'humanité (Prudence dans l'Hamartigenia vv. 395-397 mentionne une liste de vices qui constituent le cortège de Satan, Avit dans le chant II de son De spiritalis historiae gestis, II.31-32, indique par énumération les maux que la condition eschatologique fera finalement disparaitre). On pourrait rapprocher de l'opération conduite par l'auteur de la Laus seulement un passage du Centon de Proba ${ }^{34}$ qui présente, cependant, des caractéristiques bien différentes par rapport à notre passage. Avant tout, dans la Laus cette modalité de narration est un choix, là où dans le Centon, lié à l'emploi de matériels virgiliens, son utilisation est, de quelque façon, une obligation propre à ce genre poétique. Mais ce qui est plus important, dans le centon de Proba le thème de la dégradation de l'humanité est articulé selon une progression qui suit l'intertexte biblique clairement évoqué par la poétesse : ainsi la situation des protoparents expulsés de l'Eden à cause de leur péché est connotée par la rareté de la nourriture, que la nature, auparavant prodigue des biens, leur rend disponible ; la venue du règne plus dur de Jupiter après les Saturnia regna dont parle Virgile en Georgiques I, est utilisée pour indiquer la dégradation après le premier meurtre de l'humanité, celui d'Abel par Caïn ; enfin l'ultérieure dégradation qui conduit à la punition par le déluge est caractérisée comme l'âge de fer, qui est encore pire, decolor aetas selon le modèle virgilien d'Énéide VIII. En revanche dans la Laus l'image littéraire est devenue la véritable histoire et a oblitéré complétement l'histoire biblique. Ce n'est pas dû au hasard si l'auteur parle au v. 246

31 Sall. Cat. 5.8 : corrupti mores, quos... diversa inter se mala luxuria atque avaritia vexabant. Cf. aussi Cic. fin. III.75; Liv. I, praef. 11; XXXIV.4.1; Sen. epist. 7.7; 40.5; 56.5; 69.4; 90.36; dial. XI.10, 1; Tert. nat. I.5; Prud. psych. 454-457; Aug. civ. II.19.

32 Verg. Aen. VIII.324-327 : aurea quae perhibent illo sub rege fuerunt / saecula: sic placida populos in pace regebat, / deterior donec paulatim ac decolor aetas / et belli rabies et amor successit habendi. 33 Ov. met. I.128-130 : fugere pudor verumque fidesque, / in quorum subiere locum fraudesque dolique / insidiaeque et vis et amor sceleratus habendi; Avien. Arat. 338-343: ruit haec quoque rursum / vivendi series ac successere pudendo / aurea saecla habitu. Fraus ilicet et furor ardens / adque cruentus amor chalybdis.

34 Proba Cento 276-277: tum [= Gen. 3.23] victum in silvis bacas lapidosaque corna / dant rami et vulsis pascunt radicibus herbae; 290-293 : tum [= Gen. 4.8] genitor virus serpentibus addidit atris / mellaque decussit foliis ignemque removit / praedarique lupos iussit pontumque moveri / et passim rivis currentia vina repressit; 299-302 : deterior donec (= Gen. 6.4-5) paulatim ac decolor aetas, / ferrea progenies, duris caput extulit arvis / et belli rabies et amor successit habendi. / Iustitia excedens terris vestigia fecit. 
de l'offensa Dei et de la conséquente condamnation du genre humain à cause des fautes communes (v. 247 communes ... culpas) après l'énumération de tous les maux qui ont corrompu l'humanité. Il n'y a pas un moment décisif, où, comme le témoigne la Bible, l'homme se détache de Dieu, le péché originel, mais, à travers l'itération d'hinc... hinc..., la venue de la corruption généralisée est associée à la condamnation de l'homme de la part de Dieu.

Davantage encore : cette histoire littéraire remplaçant l'histoire biblique conditionne l'interprétation théologique même de la figure de Jean Baptiste. Celui-ci, donc, représente le renouveau exceptionnel, inimitable, dans des temps caractérisés par la dégradation, du modèle de vie primitive bienheureuse, ignorant du péché (v. 252 labe immunis ab omni). Pour cela cette exceptionnalité est remarquée à travers la représentation de Jean Baptiste en tant que véritable héros des temps anciens : il est vrai qu'Ambroise dans son De Helia donne du précurseur de Jésus une caractérisation très proche de notre texte, qui sans aucun doute constitue son intertexte ${ }^{35}$, en associant ses surenchères ascétiques, capables d'aller bien au-delà des limites de la condition humaine, à une interprétation de sa qualification d'angelus en Mt, non comme messager mais comme ange ${ }^{36}$, mais l'emploi au v. 252 de semideus, qui est un terme totalement inusuel dans la poésie chrétienne pour indiquer quelqu'un qui, en tout cas , appartient à la dimension humaine, de l'adjectif invictus pour le qualifier, et du verbe transgredior qui est plus fort par rapport à supergredior utilisé par Ambroise, ainsi que la mention de son labor, ne peuvent pas ne pas évoquer cette dimension héroïque. Ce qui compte le plus, ce paradigme héroïque fournit un modèle actuel aux hommes : au lieu de se concentrer sur la condition pécheresse de l'humanité due à sa corruption, le poète, sur la base de ce modèle, attire l'attention du lecteur plutôt (mirabimus immo) sur un idéal de vie adapté à l'homme même après les péchés (vel post peccata). La caractérisation, donc, de la vie de Baptiste comme reprise héroïque de la vie primitive, avant la corruption progressive des mœurs de l'humanité, sert à faire ressortir un enseignement actuel : il est encore possible, même dans cette condition pécheresse de dégradation, bien lointaine de celle des débuts de l'humanité, de poursuivre l'idéal ascétique.

35 Ambr. Helia 3.4 : in hac virtute et spiritu Heliae venit Johannes. Denique in deserto et ille vacabat ieiuniis: «esca autem eius erant locustae et mel silvestre» (Mt 3.4). Et ideo qui vitae humanae possibilitatem continentia supergressus fuerat, non homo, sed angelus aestimatus est. De ipso legimus: «Etiam plus quam propheta. Hic est de quo scriptum: Ecce mitto angelum meum ante faciem tuam qui praeparabit viam tuam ante te» (Mt 11.9-10).

36 Cette interprétation 'orientée' du mot angelus est mise à profit dans la Laus aux vv. 317-318. 


\section{Conclusions}

On peut tirer, donc, à la fin de l'analyse de ces cas, quelques conclusions qui me semblent intéressantes et susceptibles d'approfondissements ultérieurs. Observons, avant tout, que le thème littéraire païen à propos duquel on a remarqué les affirmations programmatiques et les modalités de composition les plus originales parmi les paraphrases vétéro et néotestamentaires, est celui de la préhistoire de l'humanité, de sa condition initiale et de sa décadence ou de son progrès ultérieur. Il s'agit d'un thème qui croise nécessairement le récit biblique de la perte de la condition édénique de la part de l'homme à cause du péché originel et celui de la dégradation progressive de l'humanité, et qui pour cela est inséré par certains poètes dans le tissu de la vérité biblique.

Deuxièmement, on doit remarquer que les poètes trouvent dans ce thème, pour ainsi dire, un espace 'neutre' pour employer de façon légitime les fictions de la littérature classique en tant qu'exemplaires de cette dimension de chute et de décadence, et pour discuter les grandes questions de la littérature classique, celles du progrès ou de la corruption de l'humanité, comme le fait Victorius très probablement en rapport avec la destination didactique, voire scholastique de son Alethia, ou pour transmettre des considérations théologiques à travers des formes bien compréhensibles à un auditoire cultivé, peu à l'aise avec l'exégèse biblique : en effet, la substitution du récit biblique avec l'histoire littéraire de l'humanité primitive, favorisée par la représentation orientée de la figure de Jean Baptiste, permet à l'auteur de la Laus de valoriser aux yeux de ce public l'expérience de l'anachorèse comme restauration authentique de la dimension paradisiaque et de souligner ainsi, à travers l'exemple du Précurseur du Christ, l' 'actualité' de l'idéal ascétique, montrant celuici ce que la nature humaine peut encore accomplir même dans sa condition pécheresse.

En outre, ces éléments posent le problème fascinant du contexte dans lequel ces compositions s'insèrent. J'ai étudié ce contexte à propos de l'Alethia, je vais le faire pour la Laus. Mais il me plait ici de faire remarquer que et l'Alethia et la Laus nous ont été transmises par un seul même manuscrit, le Parisinus Latinus 7558 de l'époque carolingienne, à côté d'autres compositions, comme la correspondance en vers entre Ausone et Paulin, le poème adressé encore par Paulin à Jovius pour l'inviter à ne plus se dédier à la poésie profane, mais à se consacrer à une véritable poésie chrétienne, et un petit poème appelé Epigramma Paulini : il s'agit des compositions toutes intéressées au rapport entre littérature et en général culture classique et nouveauté chrétienne, ce qui témoigne d'un choix savant-et je dirais idéologiquement orienté- de la part de celui qui a fait ce recueil, un choix qui mérite d'être attentivement étudié. 


\section{Bibliographie}

Costanza (1985): Salvatore Costanza, «Da Giovenco a Sedulio. I proemi degli Evangeliorum libri $e$ del Carmen Paschale», in: Civiltà Classica e Cristiana 6, 253-286.

Cutino (2009): Michele Cutino, L'Alethia di Claudio Mario Vittorio: la parafrasi biblica come forma di espressione teologica, Roma.

Deproost (1998): Paul-Augustin Deprooost, «Ficta et facta : la condamnation du »mensonge des poètes« dans la poésie latine chrétienne», in: Revue des Études Augustiniennes 44, 101-121.

Duval (1989): Yves-Marie Duval, «Les premiers rapports de Paulin de Nole avec Jérôme: moine ou philosophe? Poète ou exégète?», in: Studi Tardoantichi 7, 177-216

Flury (1973): Paul Flury, «Das sechste Gedicht des Paulinus von Nola», in: Vigiliae Christianae 27, $129-145$.

Lupieri (1984): Edmondo Lupieri, «Felices sunt qui imitantur Iohannem (Hier. Hom. In lo.). La figura di S. Giovanni Battista come modello di santità », in: Augustinianum 24, 33-71.

Nazzaro (2003): Antonio Vincenzo Nazzaro, «L'annunzio dell'Angelo a Zaccaria (LC. 1, 5-25) nelle parafrasi di Giovenco (I, 1-51) e Paolino di Nola (carm. 6, 27-107)», in Centro internazionale di studi sulla poesia greca e latina in età tardoantica e medievale. Quaderni 2, 19-33.

Nazzaro (2004): Antonio Vincenzo Nazzaro, «L'annunzio dell'angelo a Maria (LC. 1, 26-38) nelle riscritture metriche di Giovenco (I, 52-79) e Paolino di Nola (carm. 6, 108-138)», in Munera amicitiae. Studi di storia e cultura sulla Tarda Antichità offerti a Salvatore Pricoco, Soveria Mannelli, 283-306.

Nazzaro (2002-2006): Antonio Vincenzo Nazzaro, «La nascita di Giovanni Battista (LC. 1, 57-80) nelle riscritture metriche di Giovenco (I, 105-132) e Paolino di Nola (carm. 6, 229-235)», in: Impegno e dialogo 15, 133-146.

Nazzaro (2006-2007): Antonio Vincenzo Nazzaro, «La veste e il cibo del Battista (Mc 1, $6=$ Mt 3, 4) nelle riscritture metriche di Giovenco (I, 323-325) e Paolino di Nola (carm. 6, 179-228)», in: Rendiconti dell'Accademia di Archeologia Lettere e Belle Arti 74, 315-324.

Roberts (1980): Michael Roberts, «The Prologue to Avitus De spiritalis historiae gestis. Christian Poetry and Poetic License», in: Traditio 36, 399-407.

Turcan-Verkerk 2003: Anne-Marie Turcan-Verkerk, Un poète latin chrétien redécouvert: Latinius Pacatus Drepanius, panégyriste de Théodose, Bruxelles.

Weidmann (1991): Clemens Weidmann, «Zu des Quellen des Paulinus von Petricordia», in: Wiener Studien 104, 169-189. 
